\title{
SORIN DUMITRESCU \\ Structures géométriques sur les courbes et les surfaces complexes
}

\author{
Annales de la faculté des sciences de Toulouse $\sigma^{e}$ série, tome 10 , \\ $\mathrm{n}^{\mathrm{o}} 3$ (2001), p. 507-531 \\ <http://www.numdam.org/item?id=AFST_2001_6_10_3_507_0>
}

(C) Université Paul Sabatier, 2001, tous droits réservés.

L'accès aux archives de la revue «Annales de la faculté des sciences de Toulouse » (http://picard.ups-tlse.fr/ annales/) implique l'accord avec les conditions générales d'utilisation (http://www.numdam.org/conditions). Toute utilisation commerciale ou impression systématique est constitutive d'une infraction pénale. Toute copie ou impression de ce fichier doit contenir la présente mention de copyright.

\section{Numdam}

Article numérisé dans le cadre du programme

Numérisation de documents anciens mathématiques

http://www.numdam.org/ 


\title{
Structures géométriques sur les courbes et les surfaces complexes ${ }^{(*)}$
}

\author{
SORIN DUMitrescu ${ }^{(1)}$
}

RÉSUMÉ. - Nous classifions les structures géométriques holomorphes sur les surfaces de Riemann compactes. Nous donnons également une description assez précise des $G$-structures holomorphes sur les surfaces complexes. Nous montrons que, sur les surfaces complexes compactes, les $G$-structures holomorphes sont souvent localement homogènes.

Abstract. - We classify holomorphic geometric structures on compact Riemann surfaces. We give a rather precise description of holomorphic $G$ structures on complex surfaces. We show that, on the compact complex surfaces, these $G$-structures are very often locally homogeneous.

\section{Introduction}

Une variété complexe compacte peut posséder diverses structures géométriques holomorphes. Les tenseurs holomorphes ou les connexions affines holomorphes sont des exemples de telles structures.

Pour définir en toute généralité le concept abstrait de structure géométrique ( $A$-structure dans la terminologie de M. Gromov [11]) holomorphe associons à une variété complexe $M$ de dimension $n$ son fibré des $r$-repères (le fibré des $r$-jets de carte) [11]. Désignons-le par $R^{r}(M)$ et précisons qu'il s'agit d'un fibré principal au-dessus de $M$ de groupe structural $D^{r}\left(\mathbf{C}^{n}\right)$, où $D^{r}\left(\mathbf{C}^{n}\right)$ est le groupe des $r$-jets de germes de biholomorphismes locaux de $\mathbf{C}^{n}$ qui préservent 0 . Ce groupe porte une structure naturelle de groupe algébrique linéaire complexe.

(*) Reçu le 27 octobre 2000, accepté le 14 septembre 2001

(1) École Normale Supérieure, Département de Mathématiques et Applications, U.M.R. 8553 du C.N.R.S., 45, Rue d'Ulm, 75230 Paris Cedex 05 France.

Courriel : Sorin.Dumitrescu@ens.fr 
La définition suivante est explicite chez C. Ehresmann [9] ou M. Gromov [11], mais ce genre d'objet est en fait considéré depuis $\mathrm{S}$. Lie et $\mathrm{E}$. Cartan.

DÉFINITION 1.1. - Soit $Z$ une variété quasi-projective munie d'une action algébrique de $D^{r}\left(\mathbf{C}^{n}\right)$. Une structure géométrique holomorphe de type $Z$ et d'ordre $r$ sur la variété $M$ est une application holomorphe $D^{r}\left(\mathbf{C}^{n}\right)$ équivariante $\phi: R^{r}(M) \rightarrow Z$, i.e. telle que $\phi(s \cdot g)=g^{-1} \cdot \phi(s)$, pour tout $s$ dans $R^{r}(M)$ et pour tout élément $g$ de $D^{r}\left(\mathbf{C}^{n}\right)$.

Une structure géométrique est dite de type algébrique affine si la variété $Z$ est affine.

Une structure géométrique s'interprète également comme une section d'un fibré naturel construit au-dessus de la variété $M$. Il s'agit du fibré de fibre $Z$ associé au fibré principal $R^{r}(M)$ via l'action du groupe structural $D^{r}\left(\mathbf{C}^{n}\right)$ sur $Z$.

Dans la première partie de ce travail (section 3) nous classifions les structures géométriques holomorphes de type affine sur les surfaces de Riemann compactes (courbes). Nous prouvons que ces structures sont, pour l'essentiel, des sections holomorphes des fibrés des jets de sections d'une puissance tensorielle du fibré tangent. Ces fibrés admettent des sections non holonomes.

THÉORÈme 1. - (i) Sur les surfaces de Riemann compactes, de genre $g \geqslant 2$, les structures géométriques holomorphes, de type algébrique affine, indécomposables, sont les sections holomorphes des fibrés des jets des sections holomorphes de $K^{\otimes \alpha}, \alpha \geqslant 0$, où $K$ désigne le fibré canonique.

(ii) Sur $P^{1}(\mathbf{C})$ les structures géométriques holomorphes, de type algébrique affine, indécomposables, sont les sections holomorphes des fibrés des jets des sections holomorphes de $K^{\otimes \alpha}, \alpha \leqslant 0$, où $K$ désigne le fibré canonique.

De plus, tous ces fibrés admettent des sections holomorphes non holonomes.

(iii) Sur les courbes elliptiques, les fibrés des r-repères étant holomorphiquement triviaux, les seules structures géométriques holomorphes de type affine sont les fonctions holomorphes (constantes).

Dans la deuxième partie de cet article (section 4) nous étudions les $G$ structures holomorphe. Nous rappelons que la structure géométrique holomorphe $\phi$ est dite $G$-structure si l'image de $\phi$ dans $Z$ est une $D^{r}\left(\mathbf{C}^{n}\right)$-orbite de $Z$. 
En dimension complexe 2, nous faisons la liste des $G$-structures holomorphes d'ordre 1. Dans le cas où une surface complexe compacte possède une telle structure, nous montrons que la $G$-structure est nécessairement localement homogène :

THÉORÈME 2. - Sur les surfaces complexes compactes toutes les Gstructures holomorphes d'ordre 1 de type affine sont localement homogènes.

Nous classifions également les $G$-structures holomorphes d'ordre 2 dont l'isotropie linéaire est $G L(n, \mathbf{C})$ ou $S L(n, \mathbf{C})$ :

THÉORÈME 3. - (i). Les G-structures holomorphes d'ordre 2 dont l'isotropie linéaire est $G L(n, \mathbf{C})$ sont : les connexions projectives holomorphes sans torsion, les connexions affines holomorphes sans torsion et les 1-jets des volumes conformes holomorphes.

(ii). Les G-structures holomorphes d'ordre 2 dont l'isotropie linéaire est $S L(n, \mathbf{C})$ sont les connexions affines holomorphes unimodulaires sans torsion et les 1-jets des volumes holomorphes.

Les structures géométriques holomorphes sur les variétés complexes compactes sont souvent localement homogènes $[6],[7],[8]$. Ces résultats d'homogénéité locale appuie fortement la conjecture générale suivante :

CONJECTURE 1. - Toute G-structure holomorphe de type affine sur une variété complexe compacte est localement homogène.

Remarquons que le théorème 1 implique, en particulier, que la conjecture précédente est vraie sur les courbes. En effet, pour $g \neq 1$, toutes les sections holomorphes des fibrés des jets des sections de $K^{\otimes \alpha}$ s'annulent. En particulier, les seules courbes qui admettent des $G$-structures sont les courbes elliptiques et sur les courbes elliptiques ces structures sont des fonctions constantes, donc localement homogènes.

La conjecture précédente est non résolue dans le cas particulier des connexions affines holomorphes sans torsion sur les surfaces complexes compactes. Pour ce qui est de surfaces, nous démontrons ici que si la conjecture précédente est vraie dans le cas des connexions sans torsion, alors elle est également vraie pour toutes les $G$-structures dont l'isotropie linéaire est $G L(2, \mathbf{C})$ ou $S L(2, \mathbf{C})$.

Nous avons aussi le résultat suivant :

THÉORÈme 4. - Sur les surfaces complexes compactes, toutes les Gstructures holomorphes de type affine dont l'isotropie est connexe et distingué sont localement homogènes. 
Dans le théorème précédent, l'hypothèse faite sur le type (affine) de la structure ne peut pas être évitée. Ceci devient entièrement clair si nous considérons un plongement holomorphe d'un tore algébrique dans un espace projectif. Ce plongement est une structure géométrique (de type non affine) non localement homogène (car non constante).

En revanche, toute application holomorphe d'une variété compacte dans une variété affine est constante et donc localement homogène en tant que structure géométrique.

La construction suivante, inspirée de [10], donne un exemple plus élaboré de structure géométrique holomorphe d'ordre 1 (de type non affine) non localement homogène sur un tore complexe de dimension 2. Cet exemple montre que le théorème 2 , valable pour les $G$-structures, ne peut pas être étendu aux structures géométriques holomorphes.

Considérons un tore $T=\mathbf{C}^{2} / \Lambda$ et supposons qu'il existe une forme linéaire $\pi: \mathbf{C}^{2} \rightarrow \mathbf{C}$ qui envoie $\Lambda$ sur un réseau $\Lambda^{\prime}$ de $\mathbf{C}$. Par abus de notation nous désignons aussi par $\pi$ la projection de $T$ sur la courbe elliptique $\mathbf{C} / \Lambda^{\prime}$. Soit $f$ une fonction méromorphe non constante sur $\mathbf{C} / \Lambda^{\prime}$ et considérons sur $T$ la forme différentielle méromorphe $\tilde{\omega}=\omega+\pi^{*}(f d z)$, où $\omega$ est une forme holomorphe globale sur $T$. Il n'est pas difficile de s'assurer que $\tilde{\omega}$ est fermée et que le feuilletage holomorophe défini par $\tilde{\omega}$ en dehors de ses pôles se prolonge en un feuilletage holomorphe non singulier sur $T$ qui s'exprime en coordonnées locales :

$$
f\left(z_{1}\right) d z_{1}+d z_{2}=0 .
$$

Contrairement au feuilletage linéaire défini par $\omega$, le feuilletage donné par $\tilde{\omega}$ n'est pas homogène : il n'est pas invariant par les translations de $T$ dans la direction $\frac{\partial}{\partial z_{1}}$. Ceci montre que la structure géométrique $\phi$ obtenue par la juxtaposition de ce feuilletage et de la métrique riemannienne holomorphe plate héritée par $T$ de la métrique canonique $d z_{1}^{2}+d z_{2}^{2}$ n'est pas localement homogène.

Remarquons aussi que la structure géométrique $\phi$ n'est pas une $G$ structure car la droite tangente au feuilletage donné par $\tilde{\omega}$ est isotrope (pour la métrique riemannienne holomorphe) en certains points de $T$ et non isotrope en d'autres. 


\section{Structures géométriques holomorphes}

\subsection{Jet d'une structure géométrique}

Le but de cette section est de rassembler les propriétés des structures géométriques holomorphes dont nous aurons l'usage par la suite. Pour une présentation détaillée du sujet le lecteur pourra se référer à [5] ou bien à [3].

Considérons une structure géométrique holomorphe $\phi$ d'ordre $r$ sur une variété complexe $M$ de dimension $n$. Le $s$-ème jet $\phi^{(s)}$ de la structure $\phi$ est alors une structure géométrique sur $M$ d'ordre $r+s$. Si $\phi$ est vue comme une section d'un fibré naturel $E$ construit à partir de $M$, son $s$-jet $\phi^{(s)}$ est la section holonome du fibré des $s$-jets de sections $\Gamma^{s}(E)$ qui lui correspond. Si dans une carte locale $\phi$ est caractérisée par une application à valeurs dans $Z, \phi^{(s)}$ s'exprime dans la même carte par le $s$-jet de l'application initiale.

Remarquons que le fibré $\Gamma^{s}(E)$ au-dessus de $M$ peut avoir d'autres sections holomorphes (qui sont donc des structures géométriques sur $M$ ) qui ne sont pas de $s$-jets de sections holomorphes de $E$. Une section de $\Gamma^{s}(E)$ qui est le $s$-jet d'une section holomorphe de $E$ est dite section holonome. Le $s$-jet d'une structure géométrique correspond, par définition, à une section holonome.

Nous nous penchons à présent sur les applications qui préservent une structure géométrique $\phi: R^{r}(M) \rightarrow Z$. Comme précédemment, $M$ désigne une variété complexe de dimension $n$, munie d'une structure géométrique $\phi$ d'ordre $r$ donnée par une application $\phi: R^{r}(M) \rightarrow Z$. Tout biholomorphisme $f$ de $M$ se relève en un biholomorphisme $\tilde{f}$ du fibré des $r$-repères. Le biholomorpisme $f$ est, par définition, une isométrie de $\phi$, si $\tilde{f}$ préserve les fibres de $\phi$, c'est-à-dire $\phi \circ \tilde{f}=\phi$. L'ensemble de ces transformations est un groupe pour la composition, le groupe des isométries $I s$ de la structure géométrique.

Souvent il est plus intéressant de regarder sur $M$ une relation d'équivalence plus souple que celle donnée par les orbites du groupe Is. Pour cela nous allons inclure dans notre étude les isométries locales. Ce sont des biholomorphismes d'un ouvert de $M$ sur un autre ouvert de $M$ qui respectent la structure $\phi$ :

DÉFINITION 2.1. - Une isométrie locale de $\phi$ est un biholomorphisme entre deux ouverts de $M$ qui préserve $\phi$.

L'ensemble des isométries locales forment le pseudo-groupe $I s^{l o r}$. La situation la plus agréable apparaît quand le pseudo-groupe $I s^{l o c}$ agit tran- 
sitivement sur $M$ (i.e. avec une seule orbite). Dans ce cas nous disons que la structure $\phi$ est localement homogène. Autrement dit, la structure $\phi$ admet une unique forme locale.

\section{2. $G$-structures}

Supposons à présent que la structure géométrique holomorphe $\phi$ est une $G$-structure. Cela revient à supposer que l'image de l'application $\phi$ dans la variété quasi-projective $Z$ (voir la définition 1.1) est une $D^{r}\left(\mathbf{C}^{n}\right)$-orbite.

Désignons par $H$ le stabilisateur d'un point de cette orbite. Il s'agit d'un sous-groupe algébrique de $D^{r}\left(\mathbf{C}^{n}\right)$. L'orbite $\operatorname{Im} \phi$ (l'image de l'application $\phi)$ s'identifie alors à l'espace homogène $D^{r}\left(\mathbf{C}^{n}\right) / H$.

Nous sommes en possession d'une application holomorphe équivariante de $R^{r}(M)$ dans $D^{r}\left(\mathbf{C}^{n}\right) / H$. Ceci est équivalent à l'existence d'un sousfibré principal de $R^{r}(M)$ de groupe structural $H$ (ce sous-fibré est l'image réciproque par $\phi$ d'un point de $\operatorname{Im} \phi$ ).

Dans ce cas nous disons aussi que le groupe structural de $R^{r}(M)$ se réduit au sous-groupe $H$ ( i.e. le fibré $R^{r}(M)$ peut s'obtenir à partir d'un 1-cocycle à valeurs dans $H$ ).

DÉfinition 2.2. - Soit $M$ une variété complexe de dimension $n$ et $H$ un sous-groupe algébrique du groupe $D^{r}\left(\mathbf{C}^{n}\right)$. Une $G$-structure holomorphe d'ordre $r$ et de groupe $H$ sur la variété $M$ est un sous-fibré principal holomorphe de groupe structural $H$ du fibré $R^{r}(M)$.

Exemple. - Une connexion affine holomorphe sans torsion est une $G$ structure holomorphe d'ordre 2 (de type affine) de groupe $G L(n, \mathbf{C})[17]$.

Le résultat suivant du à C. Chevalley (voir, par exemple, [14]) assure qu'une $G$-structure au sens de la définition précédente apparait toujours comme un cas particulier de structure géométrique au sens de la définition 1.1.

ThÉORÈme 2.3 (Chevalley). - Soit $G$ un groupe algébrique et $H$ un sous-groupe algébrique de $G$. Alors il existe une représentation algébrique projective de $G$ dans un espace projectif tel que $H$ est le stabilisateur d'un point et, par conséquent, l'orbite de ce point s'identifie au quotient $G / H$.

Une structure géométrique localement homogène est en particulier une $G$-structure.

Essayons maintenant de comparer deux $G$-structures : 
DÉfINITION 2.4. - Si les $G$ - structures $\phi$ et $\phi^{\prime}$ sont données par les sous-fibrés $\mathcal{R}$ et respectivement $\mathcal{R}^{\prime}$ du fibré des r-repères de la variété $M$ et $\mathcal{R}^{\prime} \subset \mathcal{R}$, alors nous disons que $\phi$ est une relaxation de la structure $\phi^{\prime}$.

La relation de relaxation est définie pour des $G$-structures du même ordre qui vivent sur une même variété ambiante. Remarquons dans ce cas que toute isométrie locale de $\phi^{\prime}$ est automatiquement une isométrie locale de $\phi$. Si la structure géométrique $\phi^{\prime}$ est supposée localement homogène, il en sera de même pour $\phi$.

Remarquons également que si $\phi$ une $G$-structure d'ordre $r$ (vue comme un sous-fibré $\mathcal{R}$ de $R^{r}(M)$ ), alors pour tout $l$ inférieur ou égal à $r$ l'image de $\mathcal{R}$ par la projection canonique $R^{r}(M) \rightarrow R^{l}(M)$ définit une $G$-structure d'ordre $l$.

DÉFINITION 2.5. - Toute G-structure construite à partir de la structure $\phi$ par le procédé précédent est dite subordonnée à $\phi$.

Considérons la $G$-structure $\phi$ donnée par le sous-fibré $\mathcal{R}$ de $R^{r}(M)$ et supposons que $\phi$ est localement homogène. Le $s$-jet $\phi^{(s)}$ de $\phi$ est alors une $G$ structure d'ordre $r+s$ dont le groupe est formé par les $r+s$-jets isométriques de $\phi$ qui fixent un point. En général, $\phi$ est une relaxation de l'unique $G$ structure d'ordre $r$ subordonnée à $\phi^{(s)}$.

\section{Sur les surfaces de Riemann}

Dans ce chapitre nous allons nous intéresser de plus près aux structures géométriques holomorphes, de type algébrique affine, sur les surfaces de Riemann compactes.

Si $K=T^{*} C$ est le fibré canonique d'une surface de Riemann compacte connexe $C$ de genre $g \geqslant 2$, nous montrons ici que les structures géométriques holomorphes d'ordre $r$ sur $C$ sont essentiellement des sections holomorphes du fibré des $(r-1)$-jets de sections d'une puissance positive $K^{\otimes n}$ du fibré canonique.

Pour $P^{1}(\mathbf{C})$ nous avons une situation duale : il n'existe pas de formes différentielles, mais des champs de vecteurs. Il convient donc de considérer des sections du fibré des $(r-1)$-jets de sections d'une puissance négative du fibré canonique.

Le cas des tores complexes est en quelque sorte trivial, car le fibré des $r$-repères est holomorphiquement trivial. Une structures géométriques de type affine sur un tore s'exprime alors par une fonction holomorphe du tore 
à valeurs dans une variété affine. Cette fonction est nécessairement constante (il n'en est pas de même pour les structures de type non affine). Par la suite nous allons nous intéresser aux surfaces de Riemann de genre $g \neq 1$.

\subsection{Structures d'ordre 1}

Commençons par la recherche des structures géométriques holomorphes, de type algébrique affine, d'ordre 1 . Cela ne présente pas de difficulté sur les surfaces de Riemann, mais le résultat est néanmoins intéressant car il fournit des exemples de structures géométriques holomorphes qui ne sont pas localement homogènes.

Soit $C$ une surface de Riemann compacte connexe. Le groupe structural du fibré des repères $R^{1}(C)$ est $\mathbf{C}^{*}$. Afin de construire les structures géométriques holomorphes d'ordre 1 de type algébrique affine nous sommes amenés à considérer les représentations linéaires algébriques de $\mathbf{C}^{*}$ et les sections des fibrés vectoriels associés à $R^{1}(C)$ via ces représentations.

D'après un résultat classique (voir [14]), toute représentation linéaire algébrique de $\mathbf{C}^{*}$ dans un espace vectoriel $\mathbf{C}^{k}$ se diagonalise :

$$
t \cdot\left(z_{1}, \ldots, z_{k}\right)=\left(t^{\alpha_{1}} z_{1}, \ldots, t^{\alpha_{k}} z_{k}\right), \forall t \in \mathbf{C}^{*} .
$$

Le fibré vectoriel associé au fibré $R^{1}(C)$ et à la représentation précédente est une somme de fibrés en droites sur $C: K^{\otimes\left(-\alpha_{1}\right)} \oplus \ldots \oplus K^{\otimes\left(-\alpha_{k}\right)}$.

Le théorème de Riemann-Roch (voir [12], page 111) explique sous quelles conditions un fibré en droites admet des sections holomorphes :

\section{THÉORÈmE 3.1 (Riemann-Roch)}

Si $\xi$ est un fibré en droites sur la surface de Riemann compacte connexe $C$, de genre $g$, alors

$$
\operatorname{dim}^{0}(C, \xi)-\operatorname{dim} H^{1}(C, \xi)=\operatorname{deg}(\xi)+(1-g) .
$$

En particulier, si $\xi$ est une puissance entière $K^{\otimes \alpha}$, du fibré canonique, nous avons

$$
\operatorname{dim}^{0}\left(C, K^{\otimes \alpha}\right)-\operatorname{dimH}^{1}\left(C, K^{\oslash \alpha}\right)=\alpha(2 g-2)+(1-g) .
$$

Nous utiliserons aussi le théorème de dualité de Serre [12] : 
THÉORÈme 3.2 (Serre). - Si $\xi$ est un fibré en droites sur la surface de Riemann compacte $C$, alors il existe une dualité canonique entre les espaces vectoriels $\mathrm{H}^{0}(C, \xi)$ et $\mathrm{H}^{1}\left(C, K \otimes \xi^{-1}\right)$.

En particulier, $\operatorname{dim}^{0}\left(C, K^{\otimes \alpha}\right)=\operatorname{dimH}^{1}\left(C, K \otimes K^{\otimes(-\alpha)}\right)$.

Plaçons-nous d'abord dans le cas $g \geqslant 2$ et $\alpha \geqslant 1$ et remarquons que le théorème de Riemann-Roch implique que la dimension de l'espace $\mathrm{H}^{0}\left(C, K^{\otimes \alpha}\right)$ est strictement positive. Il existe donc des formes différentielles holomorphes et des puissances positives de formes différentielles holomorphes. Il n'est pas difficile de voir, à l'aide des deux théorèmes précédents, que $\operatorname{dimH}^{0}(C, K)=g$. Il existe donc sur $C$ des formes différentielles holomorphes non nulles, qui s'annulent en au moins un point. Le résultat de la spécialisation d'une telle forme sur un éventuel champ de vecteurs holomorphe est la fonction identiquement nulle. Ceci implique que le seul champ de vecteurs holomorphe sur $C$ est le champ nul. Le même raisonnement prouve qu'il n'existe pas sur $C$ des sections non nulles d'une puissance négative du fibré canonique.

Des remarques analogues montrent que, de manière duale, sur $P^{1}(\mathbf{C})$ il existe des puissances positives des champs de vecteurs et il n'existe pas de formes ou des puissances positives des formes différentielles.

Nous avons donc :

Proposition 3.3. - Toute structure géométrique holomorphe d'ordre 1 de type algébrique affine sur une surface de Riemann compacte $C$ de genre $g \geqslant 2$ (resp. $g=0$ ) est une section holomorphe d'une puissance positive du fibré canonique (fibré tangent) ou une juxtaposition d'un nombre fini de telles sections.

Remarque. - le théorème de Riemann-Roch montre que la dimension de l'espace des sections holomorphes du fibré $K^{\otimes \alpha}$ est strictement supérieure à la dimension de l'espace des sections du fibré canonique $K$. Il existe donc des sections de $K^{\otimes \alpha}$ qui ne proviennent pas de sections de $K$.

Ceci nous permet également d'exhiber des structures géométriques holomorphes qui ne sont pas localement homogènes sur aucun ouvert dense de $C$. En effet, sur $P^{1}(\mathbf{C})$, l'espace des sections holomorphes d'une puissance positive $\alpha$ du fibré tangent est un espace vectoriel complexe de dimension $2 \alpha+1$. Le "quotient" de deux éléments non nuls de cet espace vectoriel peut être considéré comme une fraction rationnelle sur $P^{1}(\mathbf{C})$. Cela montre que la structure géométrique holomorphe qui est donnée par la juxtaposition de deux sections holomorphes globales d'une puissance positive du fibré 
tangent n'est pas une structure localement homogène : on peut fabriquer l'invariant scalaire qui au point $m \in P^{1}(\mathbf{C})$ vaut la valeur en $m$ de la fraction rationnelle "quotient" des sections en question. Génériquement cette fraction rationnelle n'est pas constante et sépare les orbites du pseudo-groupe $I s^{l o c}$.

\subsection{Structures d'ordre supérieur}

Nous montrons d'abord qu'il suffit d'étudier les structures d'ordre 2. Ceci est dû au fait que toute surface de Riemann compacte admet une structure projective holomorphe. Autrement dit, il existe un recouvrement de la surface par des ouverts biholomorphes à des ouverts de $P^{1}(\mathbf{C})$ tel que les applications de changement de cartes soient des restrictions de transformations projectives de $P^{1}(\mathbf{C})$.

Nous rappelons que pour chaque 2-jet d'application de $P^{1}(\mathbf{C})$ dans luimême, il existe une unique transformation projective de $P^{1}(\mathbf{C})$ qui le réalise.

Proposition 3.4. - Soit $R^{r}(C)$ le fibré des r-repères d'une surface de Riemann compacte $C$, pour $r \geqslant 2$. Alors il existe une section holomorphe du fibré des 2-repères $R^{2}(C)$ dans $R^{r}(C)$.

\section{Démonstration}

Un résultat classique affirme que toute surface de Riemann compacte admet une structure projective (voir [12], page 172). Il existe donc sur $C$ un atlas tel que tous les changements de cartes soient projectifs. Nous appelons carte projective de $C$ une carte compatible avec cet atlas.

Nous obtenons un morphisme injectif de fibrés principaux

$$
R^{2}(C) \stackrel{i}{\rightarrow} R^{r}(C)
$$

en associant à un élément $j^{2} \in R^{2}(C)$, le $r$-jet de l'unique carte projective qui le réalise.

Remarque :

(i) La proposition précédente affirme que le groupe structural du fibré $R^{r}(C)$ admet une réduction au groupe $D^{2}(\mathbf{C})$.

Au niveau des groupes structuraux la section $R^{2}(C) \stackrel{i}{\rightarrow} R^{r}(C)$ vient avec une section $D^{2}(\mathbf{C}) \stackrel{i^{\prime}}{\rightarrow} D^{r}(\mathbf{C})$. L'image par $i^{\prime}$ de l'élément $j^{2}=(z \rightarrow$ $\left.a z+b z^{2}\right)$ de $D^{2}(\mathbf{C})$ est le $r$-jet en 0 de l'unique biholomorphisme projectif 
qui réalise $j^{2}$. Explicitement, nous avons

$$
i^{\prime}\left(j^{2}\right)=\left(z \rightarrow a z\left(1+\sum_{i=1}^{r-1} \frac{b^{i}}{a^{i}} z^{i}\right)\right) .
$$

(ii) Soit $\phi: R^{r}(C) \rightarrow Z$ une structure géométrique holomorphe de type $Z$ et d'ordre $r$. Alors l'application $\phi \circ i$ définit une structure géométrique d'ordre 2 et de type $Z$. Remarquons que cette fois $Z$ est une $D^{2}(\mathbf{C})$-variété, l'action de $D^{2}(\mathbf{C})$ se faisant à travers l'inclusion $i^{\prime}$.

Il suffit de comprendre l'interprétation géométrique de la structure (d'ordre 2) $\phi \circ i$ pour comprendre également la structure initiale $\phi$. Par exemple, si $\phi \circ i$ est une forme différentielle holomorphe sur $C$, il en est de même pour $\phi \ldots$

Nous cherchons à présent les structures géométriques d'ordre 2 de type algébrique affine. Nous commençons par identifier les représentations linéaires du groupe $D^{2}(\mathbf{C})$.

Proposition 3.5. - (i) Toute représentation linéaire algébrique indécomposable du groupe $D^{2}(\mathbf{C})$ est de la forme $\sigma_{j} \otimes T_{m}, j \in \mathbf{Z}, m \in \mathbf{N}^{*}$, où $\sigma_{j}$ est la représentation de dimension 1 , donnée par le caractère $(z \rightarrow$ $\left.a z+b z^{2}\right) \rightarrow a^{j}$ et $T_{m}$ est la représentation de dimension $m$ caractérisée par :

$$
\begin{gathered}
T_{m}((z \rightarrow a z))=\left(\begin{array}{ccccc}
1 & & & & 0 \\
& a & & \\
& & \ddots & \\
0 & & & a^{m}
\end{array}\right), \\
T_{m}\left(\left(z \rightarrow z+b z^{2}\right)\right)=\exp \left(\begin{array}{ccccc}
0 & b & 0 & \ldots & 0 \\
\vdots & 0 & \ddots & & 0 \\
\vdots & & \ddots & 0 & b \\
0 & \ldots & & & 0
\end{array}\right) .
\end{gathered}
$$

(ii) Pour $j \neq-(m-1)$, la représentation $\sigma_{j} \otimes T_{m}$ de $D^{2}(\mathbf{C})$ est obtenue via l'inclusion $D^{2}(\mathbf{C}) \stackrel{i^{\prime}}{\rightarrow} D^{r}(\mathbf{C})$, à partir de l'action canonique de $D^{r}(\mathbf{C})$ sur l'espace vectoriel de $(r-1)$-jets en 0 de:

- puissances des formes différentielles : $d z^{-j-(m-1)}$. pour $j \leqslant-m$.

- puissances des champs de vecteurs : $\left(\frac{\partial}{\partial z}\right)^{j+(m-1)}$, pour $j>-(m-2)$. 
Remarque : la proposition précédente montre que le fibré associé à $R^{2}(C)$ par la représentation $\sigma_{j} \otimes T_{m}$ de $D^{2}(\mathbf{C})$ s'identifie au fibré des jets d'ordre $(r-1)$ des sections holomorphes de $K^{-j-(m-1)}$. Les sections de ces fibrés sont les structures géométriques holomorphes d'ordre $r$.

\section{Démonstration}

(i) L'algèbre de Lie de $D^{2}(\mathbf{C})$ est de dimension 2. Elle est engendrée par les éléments $X_{1}$ et $X_{2}$, où $X_{1}, X_{2}$ sont les champs de vecteurs invariants par les translations à gauche qui correspondent respectivement aux sousgroupes à un paramètre $(t \rightarrow \exp (t) z), t \in \mathbf{C}$ et $\left(t \rightarrow z+t z^{2}\right), t \in \mathbf{C}$. Nous avons la relation $\left[X_{1}, X_{2}\right]=-X_{2}$.

Une action de $D^{2}(\mathbf{C})$ sur $\mathbf{C}^{m}$ est complètement déterminée par l'action infinitésimale de son algèbre de Lie et donc par l'action des éléments $X_{1}$ et $X_{2}$. Notons par $A \in \mathcal{M}_{m, m}(\mathbf{C})$ l'action de $X_{2}$. Il s'agit de l'action infinitésimale du sous-groupe unipotent $\left(t \rightarrow z+t z^{2}\right)$. Comme l'action est linéaire et algébrique, d'après un résultat classique [14], son image $\exp (t A)$ sera encore unipotente. La matrice $A$ est donc nilpotente.

Désignons par $B \in \mathcal{M}_{m . m}(\mathbf{C})$, l'action infinitésimale de $X_{1}$. Nous avons la relation $A B-B A=-A$. La matrice $B$ est diagonalisable car toute action linéaire et algébrique de $\mathbf{C}^{*}$ se diagonalise. La relation qui existe entre les opérateurs $A$ et $B$ implique que le noyau de $A$ est stable par $B$ et donc il existe une base de $\mathbf{C}^{m}$ dans laquelle $A$ est une matrice triangulaire supérieure avec les coefficients diagonaux nuls et $B$ est une matrice diagonale,

$$
B=\left(\begin{array}{ccc}
k_{1} & & 0 \\
& \ddots & \\
0 & & k_{m}
\end{array}\right) .
$$

La relation $A B-B A=-A$ s'exprime à l'aide des coefficients des deux matrices par $a_{s, s+l} \cdot\left(k_{l}-k_{s}+1\right)=0$. Dans le cas d'une représentation indécomposable la solution est :

$$
B=\left(\begin{array}{cccc}
k & & & 0 \\
& k+1 & & \\
& & \ddots & \\
0 & & & k+m
\end{array}\right),
$$


Structures géométriques sur les courbes et les surfaces complexes

$$
A=\left(\begin{array}{ccccc}
0 & 1 & 0 & \ldots & 0 \\
\vdots & 0 & \ddots & & 0 \\
\vdots & & \ddots & 0 & 1 \\
0 & \ldots & & & 0
\end{array}\right) .
$$

Une vérification facile montre que cette représentation infinitésimale s'intégre en la représentation linéaire du groupe $D^{2}(\mathbf{C})$ décrite à la partie (i) de l'énoncé.

(ii) La preuve se fait par calcul direct. Par exemple :

- $\sigma_{-2} \otimes T_{2}$ est l'action de $D^{2}(\mathbf{C})$ sur les 1-jets en 0 des formes différentielles. tielles.

- $\sigma_{-3} \otimes T_{3}$ est l'action de $D^{2}(\mathbf{C})$ sur les 2-jets en 0 des formes différen-

Ceci termine la preuve de la proposition.

À présent, nous devons détecter parmi ces représentations celles qui produisent des fibrés vectoriels associés à $R^{2}(C)$, admettant des sections holomorphes non identiquement nulles.

Fixons une surface de Riemann compacte $C$, de genre $g \geqslant 2$. Si la représentation $\sigma_{j} \otimes T_{m}$ permet de construire un fibré vectoriel qui admet des sections holomorphes non nulles, alors il existe, en particulier, une section holomorphe non nulle de $K^{-j-(m-1)}$ et donc nécessairement $j \leqslant-(m-1)$.

Pour $j<-(m-1)$, le fibré associé à la représentation $\sigma_{j} \otimes T_{m}$ admet des sections holomorphes évidentes qui sont les $(m-1)$-jets des sections holomorphes de $K^{-j-(m-1)}$ : les sections holonomes. Plus loin nous allons montrer que ces fibrés possèdent des sections qui ne sont pas holonomes.

Mais avant nous prouvons que pour $j=-(m-1)$, le fibré vectoriel associé à la représentation $\sigma_{j} \otimes T_{m}$ possède des sections holomorphes non nulles seulement dans un cas dégénéré qui donne une structure d'ordre 1 (cas déjà étudié).

Commençons par faire la preuve pour $m=2$, l'idée de la preuve étant la même dans le cas général. Il s'agit de considérer le morphisme de groupe $D^{2}(\mathbf{C}) \rightarrow G L(2, \mathbf{C})$ qui envoie le 2-jet $\left(z \rightarrow a z+b z^{2}\right)$ sur la matrice $\left(\begin{array}{cc}a^{-1} & b a^{-2} \\ 0 & 1\end{array}\right)$, et de voir que le fibré vectoriel associé $R^{2}(\mathbf{C}) \propto \mathbf{C}^{2}$ n'admet pas de sections holomorphes. 
Soit $U_{s}, U_{t}$ deux cartes locales de la variété $C$ sur lesquelles la coordonnée est désignée respectivement par $z_{s}$ et $z_{t}$. Nous supposons que le biholomorphisme de changement de cartes est une application non triviale $U_{t}^{\prime} \subset U_{t} \stackrel{g_{s t}}{\rightarrow} U_{s}^{\prime} \subset U_{s}$

Une section holomorphe globale du fibré $R^{2}(\mathbf{C}) \propto \mathbf{C}^{2}$ s'exprime en coordonnées locales par des fonctions $U_{s} \rightarrow \mathbf{C}^{2}, z_{s} \rightarrow\left(\begin{array}{c}X_{s}\left(z_{s}\right) \\ Y_{s}\left(z_{s}\right)\end{array}\right)$, qui se recollent d'après la règle

$\left(\begin{array}{c}X_{s}\left(g_{s t}\left(z_{t}\right)\right) \\ Y_{s}\left(g_{s t}\left(z_{t}\right)\right)\end{array}\right)=\left(\begin{array}{cc}g_{s t}^{\prime-1}\left(z_{t}\right) & \frac{1}{2} \cdot g_{s t}^{\prime \prime}\left(z_{t}\right) g_{s t}^{\prime-2}\left(z_{t}\right) \\ 0 & 1\end{array}\right) \cdot\left(\begin{array}{c}X_{t}\left(z_{t}\right) \\ Y_{t}\left(z_{t}\right)\end{array}\right), \forall z_{t} \in U_{t}^{\prime}$

Ceci est équivalent au système d'équations suivant :

$$
\left\{\begin{array}{l}
X_{s}\left(g_{s t}\left(z_{t}\right)\right) \cdot g_{s t}^{\prime}\left(z_{t}\right)-X_{t}\left(z_{t}\right)=\frac{1}{2} \cdot g_{s t}^{\prime \prime}\left(z_{t}\right) g_{s t}^{\prime-1}\left(z_{t}\right) \cdot Y_{t}\left(z_{t}\right) \\
Y_{s}\left(g_{s t}\left(z_{t}\right)\right)=Y_{t}\left(z_{t}\right)
\end{array}\right.
$$

Les fonctions $Y_{s}$ se recollent donc en une fonction holomorphe globale sur $C$. Comme $C$ est une variété compacte cette fonction est constante.

Si cette constante est nulle, les fonctions locales $X_{s}$ définissent une forme différentielle holomorphe. Notre structure sera donc une forme différentielle holomorphe, qui est une structure d'ordre 1.

Si les fonctions locales $Y_{s}$ sont toutes égales à une constante non nulle, par exemple à 1 , trouver des fonctions locales $X_{s}$ compatibles, revient à résoudre un problème cohomologique. Plus précisément, recoller les $X_{s}$ est équivalent à résoudre le 1-cocycle du faisceau des sections locales du fibré canonique qui s'exprime dans la coordonnée $z_{t}$ par $\delta_{s t}=\frac{1}{2} \cdot \frac{g_{s t}^{\prime \prime}\left(z_{t}\right)}{g_{s t}^{\prime}\left(z_{t}\right)} d z_{t}$.

L'obstruction au recollement des $X_{s}$ se trouve dans $\mathrm{H}^{1}(C, K)$, qui est canoniquement isomorphe par le théorème de dualité de Serre à $\mathrm{H}^{0}(C, K \otimes$ $\left.K^{-1}\right) \simeq \mathbf{C}$.

Montrons d'abord que $\delta_{s t}$ vérifie bien les conditions de cocycle. Nous devons montrer que $\delta_{s t}=-\delta_{t s}$ et que, sur l'intersection des trois cartes locales $U_{s}, U_{t}, U_{u}$, nous avons $\delta_{s t}+\delta_{u s}+\delta_{t u}=0$.

Pour montrer que $\delta_{s t}=-\delta_{t s}$ nous exprimons l'élément $\delta_{t s}$ en la coordonnée $z_{t}$. Comme $g_{t s}=g_{s t}^{-1}$, pour la première dérivée nous avons $g_{t s}^{\prime} \circ g_{s t}=$ $\frac{1}{g_{s t}^{\prime}}$. Nous dérivons une deuxième fois et trouvons

$$
\begin{gathered}
\left(g_{t s}^{\prime \prime} \circ g_{s t}\right) \cdot g_{s t}^{\prime}=-\frac{g_{s t}^{\prime \prime}}{\left(g_{s t}^{\prime}\right)^{2}} . \\
-520-
\end{gathered}
$$


Ceci implique que

$$
\frac{g_{t s}^{\prime \prime} \circ g_{s t}}{g_{t s}^{\prime} \circ g_{s t}}=-\frac{g_{s t}^{\prime \prime}}{\left(g_{s t}^{\prime}\right)^{2}},
$$

et finalement que $\delta_{t s}=-\delta_{s t}$.

Un calcul similaire, que nous ne reproduisons pas ici, montre que la deuxième condition de cocycle est bien satisfaite.

Le cocycle $\delta_{s t}$ est loin d'être anodin. En effet, il n'est pas difficile de s'assurer (voir [12], section 9.a) que l'annulation de $\delta_{s t} \in \mathrm{H}^{1}(C, K)$ est équivalente à l'existence d'une structure affine complexe sur $C$. Or, l'existence d'une structure affine sur $C$ implique que le fibré tangent à $C$ peut être représenté par un élément de $\mathrm{H}^{1}\left(C, \mathrm{C}^{*}\right)$ constitué par des fonctions constantes. Ceci est équivalent au fait que la première classe de Chern du fibré tangent à $C$ est nulle. Mais $c_{1}(C)=2 g-2$, ce qui implique que les seules surfaces de Riemann qui admettent des structures affines sont les tores et, de manière équivalente, le fibré $R^{2}(C) \propto \mathbf{C}^{2}$ ne possède pas de sections holomorphes (autre que des formes différentielles) si $g \neq 1$.

Dans le cas général, la même preuve montre que le fibré associé à la représentation $\sigma_{-(m-1)} \otimes T_{m}$ admet comme section holomorphe seulement les sections d'un sous-fibré vectoriel construit à partir de la représentation $\sigma_{-(m-1)} \otimes T_{(m-1)}$.

Comme sur $P^{1}(\mathbf{C})$ nous avons, par la dualité (champs de vecteurs $\Leftrightarrow$ formes différentielles), une situation symétrique, nous pouvons énoncer le résultat suivant.

THÉORÈME 1.- (i) Sur les surfaces de Riemann compactes, de genre $g \geqslant 2$, les structures géométriques holomorphes, de type algébrique affine, indécomposables, sont les sections holomorphes des fibrés des jets des sections holomorphes de $K^{\otimes \alpha}, \alpha \geqslant 0$.

(ii) Sur $P^{1}(\mathbf{C})$ les structures géométriques holomorphes, de type algébrique affine, indécomposables, sont les sections holomorphes des fibrés des jets des sections holomorphes de $K^{\otimes \alpha}, \alpha \leqslant 0$.

Remarque : il importe de s'assurer que les fibrés des jets du théorème précédent possèdent des sections holomorphes qui ne sont pas holonomes.

Prouvons ce résultat pour les fibrés des 1-jets. La preuve se généralise sans difficulté aux fibrés des jets d'ordre supérieur.

Soit $F$ le fibré des 1-jets de sections de $K^{\alpha}(\alpha \geqslant 0)$, sur une surface de Riemann compacte $C$, de genre $g \geqslant 2$. Il s'agit du fibré associé à $R^{2}(C)$ par 
la représentation $\sigma_{-\alpha-1} \otimes T_{2}$ :

$$
\left(z \rightarrow a z+b z^{2}\right) \rightarrow\left(\begin{array}{cc}
a^{-\alpha-1} & -\frac{2 b}{a^{\alpha+2}} \\
0 & a^{-\alpha}
\end{array}\right) .
$$

Nous avons une suite exacte courte de fibrés :

$$
0 \rightarrow K^{\alpha+1} \rightarrow F \stackrel{p}{\rightarrow} K^{\alpha} \rightarrow 0 .
$$

L'obstruction pour que $F$ soit une somme directe $K^{\alpha+1} \oplus K^{\alpha}$ vit dans l'espace suivant : $\mathrm{H}^{1}\left(C, \operatorname{Hom}\left(K^{\alpha}, K^{\alpha+1}\right)\right)$. Or,

$$
\begin{aligned}
\mathrm{H}^{1}\left(C, \operatorname{Hom}\left(K^{\alpha}, K^{\alpha+1}\right)\right) \simeq \mathrm{H}^{1}\left(C, K^{-\alpha} \otimes K^{\alpha+1}\right)= & \\
& =\mathrm{H}^{1}(C, K) \simeq \mathrm{H}^{0}\left(C, K \otimes K^{-1}\right) \simeq \mathbf{C} .
\end{aligned}
$$

Un calcul cohomologique simple montre que $F$ est égal à la somme directe $K^{\alpha+1} \oplus K^{\alpha}$ si et seulement si nous nous trouvons sur une surface de genre $g=1$. Comme le genre de $C$ est supérieur ou égal à deux, nous n'avons pas une somme directe des structures d'ordre 1 .

À présent nous nous intéressons aux sections de $F$. La suite exacte courte de fibrés nous fournit une suite exacte longue en cohomologie (voir [12], page 32) :

$$
0 \rightarrow \mathrm{H}^{0}\left(C, K^{\alpha+1}\right) \rightarrow \mathrm{H}^{0}(C, F) \rightarrow \mathrm{H}^{0}\left(C, K^{\alpha}\right) \rightarrow \mathrm{H}^{1}\left(C, K^{\alpha+1}\right) .
$$

Dans cette suite exacte, le dernier groupe est nul car, par le théorème de dualité de Serre, $\mathrm{H}^{1}\left(C, K^{\alpha+1}\right) \simeq \mathrm{H}^{0}\left(C, K^{-\alpha}\right)$.

Nous avons donc

$$
\operatorname{dim}^{0}(C, F)=\operatorname{dimH}^{0}\left(C, K^{\alpha}\right)+\operatorname{dim}^{0}\left(C, K^{\alpha+1}\right) .
$$

Ceci montre que la dimension de l'espace vectoriel des sections holomorphes de $F$ est strictement supérieure à la dimension de l'espace vectoriel des sections holomorphes de $K^{\alpha}$. Il existe donc des sections de $F$ qui ne sont pas holonomes.

\section{Recensement des $G$-structures}

\section{1. $G$-structures d'ordre 1}

Pour exhiber les $G$-structures d'ordre 1, sur les surfaces complexes, il suffit de trouver les sous-groupes algébriques $H^{1}$ du groupe $G L(2, \mathbf{C})$ et de préciser la signification géométrique d'une application holomorphe $G L(2, \mathbf{C})$ équivariante de $R^{1}(M)$ dans $G L(2, \mathbf{C}) / H^{1}$. 
THÉORÈME 4.1. - Sur les surfaces complexes compactes, les G-structures holomorphes d'ordre 1 sont (à revêtement fini près) :

(1) un parallélisme holomorphe.

(2) une forme volume holomorphe.

(3) deux feuilletages holomorphes non singuliers transverses $\mathcal{F}_{1}$ et $\mathcal{F}_{2}$ et une section holomorphe non singulière du fibré $S^{m}\left(T \mathcal{F}_{1}\right) \otimes S^{n}\left(T \mathcal{F}_{2}\right)$, où $T \mathcal{F}_{1}$ et $T \mathcal{F}_{2}$ désignent respectivement les fibrés tangents aux feuilletages $\mathcal{F}_{1}$ et $\mathcal{F}_{2}$ et $m$ et $n$ sont des entiers.

(4) un feuilletages holomorphe non singulier $\mathcal{F}$ et une section holomorphe non singulière d'un fibré $S^{m}(T \mathcal{F}) \otimes S^{n}(N \mathcal{F})$, où $T \mathcal{F}$ et $N \mathcal{F}$ désignent respectivement les fibrés tangent et normal au feuilletage $\mathcal{F}$ et $m$ et $n$ sont des entiers.

Toutes les structures d'ordre 1 et de type affine sont localement homogènes.

Remarque : le cas (3) contient les métriques riemanniennes holomorphes ( $m=-1$ et $n=-1$ ) et les trivialisations holomorphes du projectivisé du fibré tangent ( $m=-1$ et $n=1)$, tandis que le cas (4) contient les 1-formes holomorphes non singulières $(m=0$ et $n=-1)$ et les champs de vecteurs holomorphes non singuliers $(m=1$ et $n=0)$.

La preuve de la proposition, que nous ne reproduisons ici que partiellement, n'est pas difficile. Il suffit de trouver les sous-groupes algébriques de $G L(2, \mathbf{C})$ (à sous-groupe fini près) et d'interpréter géométriquement les réductions du groupe structural. Par exemple, le sous-groupe trivial Id correspond à un parallélisme holomorphe (trivialisation holomorphe du fibré tangent) de la surface, tandis que le sous-groupe parabolique formé par les matrices inversibles triangulaires supérieures correpond à un feuilletage holomorphe non singulier (champ de droites holomorphe). Le groupe $S L(2, \mathrm{C})$ correspond à l'existence d'une forme volume holomorphe sur la surface, tandis que le groupe $O(2, \mathbf{C})$ donne l'existence d'une métrique riemannienne holomorphe.

Passons à la question de l'homogénéité locale de ces structures. Pour ce qui est du parallélisme holomorphe, une conséquence directe du théorème de Wang [22] implique que les seules surfaces complexes compactes dont le fibré tangent est holomorphiquement trivial sont les tores. Dans (') cas le parallélisme est invariant par toutes les translations du tore : il est. en particulier, homogène. 
L'homogénéité locale d'une forme volume est un résultat local classique.

Le cas (3) est facilité par le fait qu'on connaît toutes les surfaces complexes compactes dont le fibré tangent est somme directe de deux fibrés en droites [20] (voir aussi [2], théorème $\mathrm{C}$ ). À revêtement fini près, il suffit de traiter le cas des métriques riemanniennes holomorphes et des trivialisations du projectivisé du fibré tangent.

Pour démontrer qu'une métrique holomorphe $q$ sur une surface complexe compacte $M$ est toujours localement homogène nous utilisons l'argument de la courbure sectionnelle. En effet, la donnée d'une métrique holomorphe implique, en particulier, l'existence d'une forme volume non singulière sur un revêtement double ou encore une section holomorphe qui ne s'annule pas du fibré $K^{2}$, où $K$ est le fibré canonique de $M$. Le fibré $K^{2}$ est, par conséquent, trivial.

Par ailleurs, nous fabriquons, à partir du tenseur de courbure $R$ de la métrique, le tenseur $(x, y) \rightarrow R(x, y, x, y)$. Les symétries de $R$ font que le dernier tenseur est une section du fibré (trivialisé) $K^{2}$. Cette section fournit donc une fonction qui est la courbure sectionnelle. Comme il s'agit d'une fonction holomorphe sur une variété compacte, elle est constante. Ceci implique, par un théorème local classique dû à Riemann ([23], théorème 2.4.11), l'unicité de la forme locale de la métrique $q$. Il est même connu que les seuls exemples de surfaces complexes compactes munies de métriques riemanniennes holomorphes sont les tores et leurs quotients finis [8]. Les métriques en question sont nécessairement plates.

La donnée d'une trivialisation holomorphe du projectivisé du fibré tangent à une surface $S$ est équivalente à l'existence d'un tissu holomorphe sur $S$ ou encore à la donnée de trois feuilletages holomorphes non singuliers transverses sur $S$. À l'aide de la classification des surfaces complexes compactes dont le fibré tangent est somme de deux fibrés en droites [2] (théorème $\mathrm{C}$ ) nous en déduisons que les seules surfaces $S$ qui portent un tissu holomorphe sont les tores complexes et les surfaces de Hopf. Dans les deux cas, l'existence de coordonnées globales permet de voir les trois feuilletages holomorphes comme une application holomorphe de $S$ dans le produit $P^{1}(\mathbf{C}) \times P^{1}(\mathbf{C}) \times P^{1}(\mathbf{C})$, quotienté par les symétries entre les facteurs. En effet, il suffit d'associer à chaque point de $S$ les trois droites de $\mathbf{C}^{2}$ qui sont tangentes aux trois feuilletages. Nous avons donc une application holomorphe de $S$ à valeurs dans $P^{3}(\mathbf{C}) \backslash\{\{X=Y\} \cup\{X=Z\} \cup\{Y=Z\}\}$. Une application immédiate du théorème de Chow montre que l'image est une sous-variété algébrique. Comme l'intersection avec une hypersurface est vide, le théorème de Bézout implique que l'image de $S$ est un point. Ceci force l'homogénéité locale. 
Parmi les structures géométriques du cas (4), les seules qui sont de type affine sont les sections holomorphes non singulières des fibrés $S^{n}(T S)$ qui prennent leurs valeurs dans le cône des puissances $n$-ièmes. Sur un revêtement d'ordre $n$ il s'agit donc d'un champ de vecteurs non singulier ou bien d'une 1-forme différentielle holomorphe non singulière. Dans le premier cas l'homogénéité locale est donnée par le théorème classique de redressement du flot et dans le deuxième cas elle découle du fait que sur une surface complexe compacte toutes les 1-formes différentielles holomorphes sont fermées.

Passons maintenant aux $G$-structures d'ordre supérieur.

\subsection{Structures d'ordre supérieur}

Avant d'étudier les sous-groupes de Lie de $D^{r}\left(\mathbf{C}^{n}\right)$, regardons de plus près ce groupe. Le lemme suivant donne la structure de son algèbre de Lie. Il s'agit d'un résultat classique que nous pouvons probablement attribuer à S. Lie. Pour la preuve nous renvoyons à [21] (theorem 2.1) ou au livre [1].

LEMME 4.2. - L'algèbre de Lie $g_{n}^{r}$ du groupe $D^{r}\left(\mathbf{C}^{n}\right)$ est l'espace vectoriel des $r$-jets en 0 , de champs de vecteurs holomorphes sur $\mathbf{C}^{n}$ qui s'annulent à l'origine, muni du crochet $\left[J^{r}(X), J^{r}(Y)\right]=-J^{r}([X, Y])$, où $J^{r}(X)$ désigne le $r$-jet en 0 du champ $X$.

L'application exponentielle est donnée par la formule : $\exp \left(J^{r}(X)\right)=$ $J^{r}\left(\Phi^{1}\right)$, où $J^{r}\left(\Phi^{1}\right)$ est le r-jet en 0 du temps 1 du flot $\Phi^{t}$ engendré par le champ $X$.

L'algèbre de Lie $g_{n}^{r}$ admet donc une décomposition naturelle

$$
g_{n}^{r}=g_{n}^{(0)} \oplus g_{n}^{(1)} \oplus \ldots \oplus g_{n}^{(r-1)},
$$

où $g_{n}^{(i)}$ est l'espace vectoriel des champs de vecteurs polynômiaux homogènes de degré $i+1$. En particulier, $g_{n}^{(0)}$ est $g l(n, \mathbf{C})$. Le crochet de Lie vérifie $\left[g_{n}^{(i)}, g_{n}^{(j)}\right] \subset g_{n}^{(i+j)}$.

DÉFInITION 4.3. - Si $H$ est un sous-groupe de $D^{r}\left(\mathbf{C}^{n}\right)$. l'image de $H$ par la projection canonique $D^{r}\left(\mathbf{C}^{n}\right) \rightarrow D^{1}\left(\mathbf{C}^{n}\right)$ est appelée la partie linéaire de $H$.

DÉFInITION 4.4. - Soit $\phi$ une G-structure de groupe H. Nous disons que $H$ est l'isotropie de $\phi$ et que la partie linéaire de $H$ est l'isotropie linéaire de la structure $\phi$. 
Passons à présent à la preuve du théorème 4 :

\section{Démonstration}

La preuve est basée essentiellement sur un résultat de [21] (theorem 2.5) qui affirme que les sous-groupes connexes et distingués de $D^{r}\left(\mathbf{C}^{n}\right)$ sont conjugués à des sous-groupes de $G L(n, \mathbf{C})$ (il existe une unique copie de $G L(n, \mathbf{C})$ dans $D^{r}\left(\mathbf{C}^{n}\right)$, celle donnée par la section de $D^{1}\left(\mathbf{C}^{n}\right)$ dans $D^{r}\left(\mathbf{C}^{n}\right)$ obtenue en associant à chaque 1-jet le $r$-jet de l'unique application linéaire qui le représente) ou ont une partie linéaire triviale.

Comme sur les surfaces complexes compactes toutes les $G$-structures d'ordre 1 sont localement homogènes (proposition 4.1), il nous reste à traiter le cas où l'isotropie linéaire est triviale. Dans cette situation la $G$-structure d'ordre 1 subordonnée à notre structure est un parallélisme et, par le théorème de Wang [22], la surface est un quotient d'un groupe de Lie complexe connexe et simplement connexe par un réseau co-compact. Or, une telle surface est nécessairement un tore (le groupe affine n'admet pas de réseau co-compact). La $G$-structure étant de type affine, elle est caractérisée par une fonction holomorphe du tore dans une variété affine. Une telle fonction est nécessairement constante et la $G$-structure en question est homogène (invariante par toute translation).

Passons à présent au cas où l'isotropie linéaire est grande.

Le théorème suivant décrit les $G$-structures holomorphes d'ordre 2 dont l'isotropie linéaire est $G L(n, \mathbf{C})$ ou $S L(n, \mathbf{C})$.

THÉORÈme 3. - (i). Les G-structures holomorphes d'ordre 2 dont l'isotropie linéaire est $G L(n, \mathbf{C})$ sont : les connexions projectives holomorphes sans torsion, les connexions affines holomorphes sans torsion et les 1-jets des volumes conformes holomorphes.

(ii). Les $G$-structures holomorphes d'ordre 2 dont l'isotropie linéaire est $S L(n, \mathbf{C})$ sont les connexions affines holomorphes unimodulaires sans torsion et les 1-jets des volumes holomorphes.

Remarque : il est classiquement connu que les formes volumes et les volumes conformes sont des structures localement homogènes.

Démonstration

Nous commençons par quelquess considérations de portée plus générale que notre énoncé. 
Soit $M$ une variété complexe munie de la $G$-structure d'ordre $r$,

$$
R^{r}(M) \stackrel{\phi}{\rightarrow} D^{r}\left(\mathbf{C}^{n}\right) / H^{r}
$$

Pour avoir les sous-groupes de Lie $H^{r} \subset D^{r}\left(\mathbf{C}^{n}\right)$ nous cherchons les sousalgèbres de Lie de $g_{n}^{r}$. Un lemme de Whitehead (voir, par exemple, [13], chapitre VII.6) assure que toute sous-algèbre de Lie de $g_{n}^{r}$ dont la partie linéaire est $g l(n, \mathbf{C})$ ou $\operatorname{sl}(n, \mathbf{C})$ est graduée, autrement dit l'algèbre de Lie $h_{n}^{r}$ de $H^{r}$ est de la forme

$$
h_{n}^{r}=h_{n}^{(0)} \oplus h_{n}^{(1)} \oplus \ldots \oplus h_{n}^{(r-1)},
$$

où $h_{n}^{(i)}$ est un sous-espace vectoriel de $g_{n}^{(i)}$.

(i). Commençons par le cas où l'isotropie linéaire est $g l(n, \mathbf{C})$. Considérons le sous-groupe $H^{r}$ de $D^{r}\left(\mathbf{C}^{n}\right)$ et la décomposition naturelle de son algèbre de Lie

$$
h_{n}^{r}=h_{n}^{(0)} \oplus h_{n}^{(1)} \oplus \ldots \oplus h_{n}^{(r-1)},
$$

$h_{n}^{(0)}=g l(n, \mathbf{C})$.

La représentation adjointe de $D^{r}\left(\mathbf{C}^{n}\right)$ sur son algèbre de Lie

$$
g_{n}^{r}=g_{n}^{(0)} \oplus g_{n}^{(1)} \oplus \ldots \oplus g_{n}^{(r-1)},
$$

fournit en restriction à sa partie linéaire une représentation de $G L(n, \mathbf{C})$ sur $g_{n}^{r}$ qui admet les espaces vectoriels $g_{n}^{(i)}$ comme espaces stables (ceci se traduit de manière infinitésimale par $\left.\left[g_{n}^{(0)}, g_{n}^{(i)}\right] \subset g_{n}^{(i)}\right)$.

Chaque espace vectoriel $g_{n}^{(i)}$ se décompose sous l'action de $G L(n, \mathbf{C})$ en deux sous-espaces irréductibles stables

$$
g_{n}^{(i)}=g_{n, R}^{(i)} \oplus g_{n, D}^{(i)}
$$

où $g_{n, R}^{(i)}$ est le sous-espace vectoriel de $g_{n}^{(i)}$ formé par des champs de vecteurs radiaux et $g_{n, D}^{(i)}$ est le sous-espace vectoriel des champs de divergence nulle. Un élément de $g_{n, R}^{(i)}$ est de la forme $P\left(z_{1}, \ldots, z_{n}\right)\left(z_{1} \frac{\partial}{\partial z_{1}}+\ldots+z_{n} \frac{\partial}{\partial z_{n}}\right)$, où $P$ est un polynôme homogène de degré $i$, en les variables $\left(z_{1}, \ldots, z_{n}\right)$.

Pour prouver que $g_{n}^{(i)}=g_{n, R}^{(i)} \oplus g_{n, D}^{(i)}$, nous observons d'abord que l'image de l'opérateur divergence, $\operatorname{div}: g_{n}^{(i)} \rightarrow C^{a n}\left(\mathbf{C}^{n}\right)$ est l'espace vectoriel des polynômes homogènes de degré $i$ en les variables $\left(z_{1}, \ldots, z_{n}\right)$. L'image est 
donc un espace vectoriel isomorphe à $g_{n, R}^{(i)}$. Comme le noyau de l'opérateur divergence est justement $g_{n, D}^{(i)}$, nous avons $\operatorname{dim} g_{n, R}^{(i)}+\operatorname{dim} g_{n, D}^{(i)}=\operatorname{dim} g_{n}^{(i)}$.

Par ailleurs, la relation d'Euler montre que la divergence d'un champ de vecteurs radial $P\left(z_{1}, \ldots, z_{n}\right)\left(z_{1} \frac{\partial}{\partial z_{1}}+\ldots+z_{n} \frac{\partial}{\partial z_{n}}\right), P$ étant homogène de degré $i$, vaut $(i+n) P$. Donc $g_{n, R}^{(i)} \cap g_{n, D}^{(i)}=\{0\}$.

Pour une preuve du fait que les représentations $g_{n, R}^{(i)}$ et $g_{n, D}^{(i)}$ sont irréductibles voir [21].

Il est facile de vérifier que $\left[g_{n, R}^{(i)}, g_{n, R}^{(j)}\right] \subset g_{n, R}^{(i+j)}$ et que $\left[g_{n, D}^{(i)}, g_{n, D}^{(j)}\right] \subset$ $g_{n, D}^{(i+j)}$.

Comme l'algèbre de Lie $h_{n}^{r}$ est stable par des crochets avec des éléments de $h_{n}^{(0)}=g l(n, \mathbf{C})$, nous avons quatre choix possibles pour chaque espace $h_{n}^{(i)}$ :

$$
h_{n}^{(i)} \in\left\{0, g_{n, R}^{(i)}, g_{n, D}^{(i)}, g_{n, R}^{(i)} \oplus g_{n, D}^{(i)}\right\} .
$$

Pour les structures d'ordre 2 nous avons les possibilités suivantes :

- $h_{n}^{(1)}=g_{n, R}^{(1)} \oplus g_{n, D}^{(1)}=g_{n}^{(1)}$. Dans ce cas la structure est triviale.

- $h_{n}^{(1)}=g_{n, D}^{(1)}$. Il s'agit du 1-jet d'un volume conforme.

- $h_{n}^{(1)}=\{0\}$.

Dans ce cas nous avons une application $D^{2}\left(\mathbf{C}^{n}\right)$-équivariante

$$
R^{2}(M) \stackrel{\bar{\phi}}{\rightarrow} D^{2}\left(\mathbf{C}^{n}\right) / G L(n, \mathbf{C})
$$

autrement dit une connexion affine holomorphe sans torsion sur $M$ [17].

- $h_{n}^{(1)}=g_{n, R}^{(1)}$.

Dans ce cas il s'agit d'une connexion projective holomorphe sans torsion (quitte à remplacer le fibré tangent à $M$ par son projectivisé, nous nous trouvons dans une situation analogue à celle décrite au point précédent).

(ii). Si le groupe d'isotropie linéaire est $S L(n, \mathbf{C})$ nous avons la même décomposition en composantes irréductibles de $g_{n}^{(i)}$, sous l'action de $h_{n}^{(0)}$. La preuve est similaire. 
Intéressons-nous de plus près aux connexions affines holomorphes et aux connexions projectives holomorphes sur les surfaces complexes compactes. Dans les articles [15], [18], [19] les auteurs montrent que les surfaces complexes compactes admettant une connexion affine holomorphe ou une connexion projective holomorphe sont minimales : elles ne contiennent aucune copie de $P^{1}(\mathbf{C})$ d'autointersection -1 . Il est prouvé dans [15] que toute surface complexe compacte admettant une connexion affine holomorphe est, à revêtement fini près, un tore complexe, une surface de Kodaira primaire, une surface de Hopf affine, une surface d'Inoue ou un fibré principal holomorphe en courbes elliptiques sur une surface de Riemann de genre $g \geqslant 2$ de premier nombre de Betti impaire. Toutes ces surfaces possèdent aussi une structure affine. Parmi les surfaces de la liste précédente, seules les surfaces d'Inoue et les fibrés principaux holomorphes en courbes elliptiques sur une surface de Riemann sont susceptibles de posséder des connexions affines holomorphes non plates. Nous ne savons pas encore prouver que toutes ces connexions sont localement homogènes.

De même, toute surface complexe compacte admettant une connexion projective holomorphe admet aussi une structure projective [18], [19]. Dans le travail [16], B. Klingler classifie les structures affines holomorphes et les structures projectives holomorphes sur les surfaces complexes compactes. Nous démontrons ici le résultat suivant :

Proposition 4.5. - Soit $M$ une surface complexe compacte telle que toutes les connexions affines holomorphes sans torsion et toutes les connexions projectives holomorphes sans torsion sont localement homogènes sur $M$. Alors toute $G$-structure dont l'isotropie linéaire est $G L(2, \mathbf{C})$ ou $S L(2, \mathbf{C})$ est localement homogène sur $M$.

Démonstration . - Considérons sur $M$ une $G$-structure dont l'isotropie est le sous-groupe $H^{r}$ de $D^{r}\left(\mathbf{C}^{2}\right)$. Supposons que l'isotropie linéaire est $G L(2, \mathbf{C})$ (la preuve étant analogue dans le cas de $S L(2, \mathrm{C})$ ).

Soit

$$
h_{2}^{r}=h_{2}^{(0)} \oplus h_{2}^{(1)} \oplus \ldots \oplus h_{2}^{(r-1)},
$$

où $h_{2}^{(i)}$ est un sous-espace vectoriel de $g_{2}^{(i)}$, la décomposition de l'algèbre de Lie de $H^{r}$. Nous avons vu que

$$
h_{2}^{(i)} \in\left\{0, g_{2, R}^{(i)}, g_{2, D}^{(i)}, g_{2, R}^{(i)} \oplus g_{2, D}^{(i)}\right\} .
$$

Nous avons quatre choix possibles pour $h_{2}^{(1)}$ : 
- $h_{2}^{(1)}=g_{2, R}^{(1)} \oplus g_{2, D}^{(1)}=g_{2}^{(1)}$. Dans ce cas la stabilité de $h_{2}^{r}$ par crochet de Lie implique $h_{2}^{(i)}=g_{2}^{(i)}, \forall i \leqslant r-1$. Nous avons donc $H^{r}=D^{r}\left(\mathbf{C}^{n}\right)$ et il s'agit de la structure triviale. Elle est localement homogène.

- $h_{n}^{(1)}=g_{n, D}^{(1)}$. L'unique structure d'ordre 2 qui est subordonnée à la $G$-structure initiale est le 1-jet d'un volume conforme qui est une structure localement homogène.

La stabilité par crochet de Lie impose $g_{2, D}^{(i)} \subset h_{2}^{(i)}, \forall i \leqslant r-1$. Notre structure est donc une relaxation d'un $(r-1)$-jet d'un volume conforme. Elle est, par conséquent, localement homogène.

- $h_{2}^{(1)}=\{0\}$. L'unique structure d'ordre 2 qui est subordonnée à notre structure est une connexion affine holomorphe sans torsion. Par hypothèse cette connexion est localement homogène. Il est facile de voir que notre structure est une relaxation du jet d'ordre $(r-2)$ de cette connexion et est donc localement homogène.

- $h_{2}^{(1)}=g_{2, R}^{(1)}$. L'unique structure d'ordre 2 qui est subordonnée à la structure initiale est une connexion projective holomorphe sans torsion. Nous concluons comme dans le cas précédent.

Ceci termine la preuve de la proposition.

\section{Bibliographie}

[1] Albert (C.) et Molino (P.). - Pseudogroupes de Lie transitifs I, Hermann, Paris, (1984).

[2] Beauville (A.). - Complex manifolds with split tangent bundle, Complex Analysis and Algebraic Geometry, Walter de Gruyter, (2000), pp 61-70.

[3] Benoist (Y.). - Orbites des structures rigides (d'après M. Gromov), Feuilletages et systèmes intégrables (Montpellier, 1995), Birkhäuser Boston, (1997), pp. 1-17.

[4] Bosio (F.). - Actions holomorphes et localement libres de groupes de Lie abéliens, Thèse, ENS-Lyon, (1996).

[5] D'Ambra (G.) et Gromov (M.). - Lectures on transformations groups: geometry and dynamics, Surveys in Differential Geometry (Cambridge), (1990), pp. 19-111.

[6] Dumitrescu (S.). - Structures géométriques holomorphes, Thèse ENS-Lyon, (1999).

[7] Dumitrescu (S.). - Structures géométriques holomorphes sur les variétés complexes compactes, Annales Scientifiques de l'ENS, $4^{\mathrm{e}}$ série, 34 (2001), pp. 557-571.

[8] Dumitrescu (S.). - Métriques riemanniennes holomorphes en petite dimension, à paraître aux Annales de l'Institut Fourier.

[9] Ehresmann (C.). - Structures locales, Ann. Math. Pur. Appl. IV, 36, (1954), pp. 133-143. 
Structures géométriques sur les courbes et les surfaces complexes

[10] Ghys (E.). - Feuilletages holomorphes de codimension un sur les espaces homogènes complexes, Annales Fac. Sci. Toulouse, V(3), (1996), pp. 493-519.

[11] Gromov (M.). - Rigid transformation groups, Géométrie Différentielle, Travaux en cours, Hermann, Paris, 33, (1988), pp. 65-141.

[12] Gunning (R.). - Lectures on Riemann Surfaces, Princeton Mathematical Notes, (1966).

[13] Hilton (P.) et Stammbach (U.). - A Course in Homological Algebra, SpringerVerlag, (1971).

[14] Humpreys (J.). - Linear algebraic groups, Graduate Texts in Mathematics 21, Springer-Verlag, (1975).

[15] Inoue (M.), Kobayashi (S.) et Ochiai (T.). - Holomorphic affine connections on compact complex surfaces, J. Fac. Sci. Univ. Tokyo Sect. IA Math., 27(2), (1980), pp. 247-264.

[16] Klingler (B.). - Structures affines et projectives sur les surfaces complexes, Ann. Inst. Fourier, 48(2), (1998), pp. 441-477.

[17] Kobayashi (S.). - Transformation Groups in Differential Geometry, SpringerVerlag, (1972).

[18] Kobayashi (S.) et OCHIAI (T.). - Holomorphic projective structures on compact complex surfaces, Math. Ann., 249(1), (1980), pp. 75-94.

[19] Kobayashi (S.) et OChiaI (T.). - Holomorphic projective structures on compact complex surfaces II, Math. Ann., 255(4), (1981), pp. 519-521.

[20] Kobayashi (S.) et Ochiai (T.). - Holomorphic structures modeled after hyperquadrics, Tôhoku Math. Journ., 34, (1982), pp. 587-629.

[21] TERNG (C.). - Natural vector bundles and natural differential operators, Amer. J. Math., 100(4), (1978), pp. 775-828.

[22] Wang (H.). - Complex parallisable manifolds, Proc. Amer. Math. Soc., 5, (1954), pp. $771-776$.

[23] Wolf (J.). - Spaces of constant curvature, McGraw-Hill Series in Higher Math., (1967). 